INTERNATIONAL JOURNAL OF MULTidisciplinARY RESEARCH AND ANALYSis

ISSN(print): 2643-9840, ISSN(online): 2643-9875

Volume 04 Issue 10 October 2021

DOI: 10.47191/ijmra/v4-i10-25, Impact Factor: 6.072

Page No.- $1496-1500$

\title{
Vitamin D Supplementation: A Potential Prevention Method against Contagious Viral Respiratory Illnesses
}

\author{
Michael Ausi, MD ${ }^{1}$, Shivaughn Hem-Lee-Forsyth, Ph.D., MPH ${ }^{2}$ \\ ${ }^{1,2}$ Department of Public Health and Preventive Medicine, St. George's University
}

\begin{abstract}
This narrative review explores the protective value of Vitamin D (VD) and its metabolites, such as Vitamin $\mathrm{D}_{2}\left(\mathrm{VD}_{2}\right)$ and Vitamin $D_{3}\left(V D_{3}\right)$, and their preventive health potentials backed by evidence-based medicine against viral respiratory illnesses (VRI). With the recent events of late 2019, a severe acute respiratory illness began to sweep the globe. This SARS illness was of coronavirus-2 variant known as SARS-CoV-2 (COVID-19). With COVID-19 in mind, the purpose of this paper was to explore a preventative health model utilizing evidence-based medical research to safeguard against similar viral catastrophes. The knowledge gained is essential for the future education of the general population and necessary for a more preemptive and protective approach to VRIs.
\end{abstract}

Google Scholar, PubMed, and DuckDuckgo were the most often utilized internet search engines and databases to collect sources. Twelve evidence-based medical articles were selected based on specific inclusionary and exclusionary criteria. More specifically, eleven systematic-review and meta-analyses (SRMA) and one Randomized Controlled Trial (RCT) were selected. The data extracted from the eleven articles were analyzed to understand the relationship between VD, VD3, and Viral Respiratory illnesses (VRIs).

VD is known to have various protective actions on the immune system (IS). First, VD activates gene transcription upon innate immune cells to increase viral defensive proteins such as Defensins and Cathelicidins (Laaski, 2011; Liu, 2006; NIH, 2020). More importantly, VD down-regulates certain specific adaptive immune cells named T helper 2 ( $\mathrm{Th}_{2}$ ). The $\mathrm{Th}_{2}$ cellular pathway indirectly downregulates T helper $1\left(\mathrm{Th}_{1}\right)$, cell pro-inflammatory cytokine storms, thus assisting with viral clearance (Huang, Wang, Li et al., 2020). More EBM research displays an inverse relationship between VD status and VRI, like incidences, severity, and duration of VRIs (Abioye, Bromage \& Fawz 2021).

Recommendations for future research were presented to understand VD better and its possible use as a potential therapeutic or preventive medicine modality for the public. Proposals on health policy changes within health agencies like the United States Preventative Task Force (USPTF) were also provided. Changes included mandatory health VD screens tables and their metabolites for patients of different ages and breeds to achieve proper VRI protection to prevent future pandemics.

KEY TERMS: Calciferol deficiency, Vitamin D, Vitamin D3, viral respiratory illness, COVID-19 pandemic, preventative health

\section{INTRODUCTION}

The world was introduced to a novel respiratory virus known as COVID-19 from November 2019 and onward. As COVID-19 made its way worldwide, the total number recorded a COVID-19, or COVID-19 complicated death toll has nearly reached $\sim 4.4$ million people worldwide according to the world meter (C. U. Live). Although, the arguments centering on public health fiscal allocations during a pandemic usually revolve around vaccine distribution, hospital therapeutic methods, and proper healthcare supplies. There has been promising research entailing a proper public health prevention method against VRIs (and their pandemics preemptively). 216 COVID-19 patients and 197 healthy individuals had VD blood levels checked in a Spanish retrospective casecontrol trial. Not only were the mean VD blood levels lower in the COVID-19 group: $13.8+/-7.2 \mathrm{ng} / \mathrm{ml}$ at $95 \%$; $82.2 \%$ of the group were VDD compared to the healthy group means $20.9+/-7.4 \mathrm{ng} / \mathrm{ml}$ and $47.2 \%$ deficient at $95 \%$, P-value $<0.0001$ for both groups (Hernández, J. L., Nan, D., \& Fernandez-Ayala, M. 2020, October 27). The main takeaway was that a high mortality rate in COVID19 patients was observed to have VDD.

Calciferol or VD is a fat-soluble vitamin, but it has the structure of a hormone. The metabolism starts with sources including fatty fish, mushrooms, eggs, sunlight exposure, and dietary supplementation. As a bolus of calciferol enters the body, it 


\section{Vitamin D Supplementation: A Potential Prevention Method against Contagious Viral Respiratory Illnesses}

undergoes an enzymatic process called hydroxylation within the liver to create $\mathrm{VD}_{2}$ or ergocalciferol. As ergocalciferol enters the bloodstream, it will eventually end up in the kidneys. Ergocalciferol is hydroxylated to become the most active metabolite of VD called $\mathrm{VD}_{3}$ or Cholecalciferol. The synthetic name is calcitriol (Sizar, 2020). Another priceless method of ensuring proper VD 3 status is to soak in fifteen minutes of sunlight. This act provides $\mathrm{VD}_{3}$ instantly by ultraviolet sunray, causing biochemical conversion of cholesterols within the skin into $\mathrm{VD}_{3}$ (Sizar, 2020). $\mathrm{VD}_{3}$ is involved in other organ systems such as the musculoskeletal, cardiovascular, central nervous, and endocrine systems (Sizar, 2020). VD and its metabolites preventative abilities lie within its physiological relationship to the IS. Viral immunity begins with $\mathrm{VD}_{3}$ manipulation of the IS's, cellular innate or adaptive immune systems (CII or CAI).

The literature has demonstrated the effectiveness of VD in a primary and tertiary prevention role against certain viral respiratory infections, virulence, and clearance (Grant, 2020). This evidence was demonstrated by supplementing proper levels of VD to balance the IS. The formulated hypothesis questioned the relationship between VD or metabolites blood levels and communicable, transmissible VRIs. VD status and VRI incidences displayed a statistically significant inverse relationship, making VD a perfect preventative measure for the general or global population.

\section{METHODS}

In this narrative review, internet database search engines such as Google, DuckDuckGo, and Google Scholar were performed. Wikipedia, PubMed, NIH, NCBI, ScienceDirect, Healthline, JAMA, MDPI, BMJ, Elsevier Health, and Cochrane Library. The internet searches started in October 2020 and concluded in July of 2021. As the headings were entered in the search engines, twenty-five articles were chosen between January 1, 2015, to July 5, 2021. MeSH Keywords: Vitamin D, Vitamin D3, COVID-19, Respiratory Virus, acute, chronic, Systematic, Review, Meta-analysis, Randomized, Controlled and trials.

Initially, twenty-five articles were selected, the researcher reviewed the titles of the SRMA and RCTs found including abstracts, and conclusions at first. Then these EBM pieces were put through an official selection process, primarily based on Inclusionary criteria: a.) Viral, respiratory, deficiency and Vitamin D was in the title, b.) review of the abstract and conclusion was done to ensure articles had preventive, treatment, management implications, and c.) the articles had to be conducted between the years 2011-2021.

The fifteen SMRA and RCT articles selected were put through a second selection review, focusing on the Exclusionary criteria list: a.) less than thirty citations from other sources, b.) RCT then sample size $<100$, c.) SRMA had $<10$ articles analyzed, and sample size $<1000$. These criteria brought a final twelve articles (eleven SRMA and one RCT) that were thoroughly assessed. The data collected from all sources were combined to analyze if VDD and VRIs have a correlation or a possible threat of causation. This analysis assisted in understanding VD's role in immunology, specifically against virology.

\section{DISCUSSION}

A combination of SRMAs and one RCT evaluated VD and its relationship with VRIs and its effects on the IS. There were eleven total SRMAs and an RCT that not only demonstrated this. First, there is a brief on VD's modulation of the IS involving the physiological association. They were followed by different VDD and metabolite deficient correlation to possible causation to many different types of VRIs in different age groups. They explicitly highlighted the relationships of VD supplementation against acute VRI's, pediatric VD concentrations against acute VRI's, and VD effect against COVID-19.

VD's role in Cellular Innate Immune homeostasis is shown directly and indirectly with the CII and CAI systems. Concerning the $\mathrm{Cll}$, viral protection starts when $\mathrm{VD}_{3}$ activates its receptors on $\mathrm{Cll}$ white blood cells to increase defense proteins called Cathelicidins (Liu, 2006) and Defensins (Laaski, 2011) via upregulating defense protein gene transcription, which is known in the literature to have antimicrobial activities against the whole spectrum of microbes including most viruses.

VD provides viral protection in cellular adaptive immune homeostasis. The CAI is part of a greater inflammatory system that is autoregulatory. When inflammation is needed for wound repair or foreign object detection, Th ${ }_{1}$ cells activate a cytokine storm called a pro-inflammatory phase. $\mathrm{VD}_{3}$ mediates anti-inflammatory or, $\mathrm{Th}_{2}$ cellular response proteins to control $\mathrm{Th}_{1}$ natural inflammatory reaction. Thus, losing Th 2 anti-inflammatory proteins can disrupt the IS' Inflammation response. COVID-19 patients were observed to have a constant Th $\mathrm{h}_{1}$ cytokine storm (Huang et al., 2020). In a double-blinded RCT, a group of Ulcerative Colitis patients was divided into two groups: those who received a single injection of VD or a placebo. After three months, the UC patients had their VD and other immunochemistry serum. VD levels were improved in the VD injection group; more importantly, it reduced pro-inflammatory $T h_{1}$ cytokine proteins such as tumor necrosis factor-alpha and interferon-gamma. The injection group also had increased production of Th2 anti-inflammatory cytokines (Shariff, Vahedi \& Nedjat, 2019). Additionally, VD and its metabolites primarily decrease interleukin-2 and secondarily promote cytokine production of $\mathrm{Th}_{2}$, enhancing the indirect suppression of $\mathrm{Th}_{1}$ 


\section{Vitamin D Supplementation: A Potential Prevention Method against Contagious Viral Respiratory Illnesses}

cells by controlling multiple multitudes of white blood cells (Cantorna, 2015). Therefore, the human body solely depends on the IS to "pass the virus" and develop an immunity to it in case of future infections.

Understanding the relationship between VD Supplementation versus Acute VRIs begins to paint the preventative health picture. By looking at the first SRMA to study VD status and VRI reviewed 25 RCTs (totaled 10,933 sample size) demonstrated VD supplementation reduced the risk of acute VRI with an adjusted Odds Ratio (OR) of $0.88+/-0.81$ to 0.96 at $95 \% \mathrm{Cl}, \mathrm{p}$-value $<0.001$ (Martineau, 2017). VD patients that took VD daily or weekly saw the greatest protective effects against acute VRIs with OR of 0.81 $+/-0.72$ to 0.91 at $95 \% \mathrm{Cl}$ (Martineau, 2017). The findings favor VD supplementation before, during, or even after a viral illness is safe and will prevent and protect against acute respiratory. The next SRMA comprised of 15 RCTs found that the patients in their studies that had VD supplementation versus placebo showed significantly lower incidences of acute VRIs with an odds ratio of $0.92+/-0.86$ to 0.99 at $95 \% \mathrm{Cl}$ (Jolliffe, Camargo, \& Sluyter 2021). To lastly add-on to the notion of VD supplementation, another SRMA done by BMJ Global Health found that not only did supplementation reduce the risk for Acute VRIs (Risk Ratio (RR) $=0.97$ $+/-0.94$ to 1.00 at $95 \%$ C.I. $p=.0028$ ) but it also shortens the duration of symptoms of acute VRIs (Absolute Risk Reduction (ARR) $=-6 \%=-9 \%$ to $-2 \%$ at $95 \% \mathrm{Cl}, \mathrm{p}=0.003$ (Abioye, et al. 2021).

Since children are viral carriers in most viral illnesses, understanding pediatric VD blood levels could potentially halt a viral spread. To begin, one SRMA found an inverse relationship between the acute VRI called, Respiratory Syncytial Virus (RSV) and VD concentration by exhibiting low VD concentration was associated with a more prolonged RSV duration of disease found in $7 / 10$ studies (Pham, Rahman \& Majidi 2019). This occurence was also confirmed by a South African SRMA that studied 409 African children aged 12 to 15 weeks, with a genetic disposition to having lower VD receptors had a higher chance of developing RSV (Laaski, 2011). Participants that had higher VD levels were protected against influenza and a core study with nearly 7000 participants.

The third pediatric SRMA focused on 12 studies that enrolled roughly 2300 pediatric participants to understand the relationship between VRIs in the Lower Respiratory Tract (LRT) and VD. Children with VRIs in the LRT had significantly lower mean VD blood levels compared to the controls. In other words, there could be a correlation between VD blood levels and the severity, duration, and incidence of acute VRIs in the LRT (Jat, 2017). The Last pediatric SRMA was done on roughly 498 patients with an average age of 19 years old were placed in a $\mathrm{VD}_{3}$ and gargling, a placebo and gargling group, and lastly, groups 3 and 4 where $\mathrm{VD}_{3}$ and Placebo alone. Recipients randomized to receive $\mathrm{VD}_{3}$ reported less clinical upper VRIs complete analysis of $R R=0.79+/-0.61$ to 1.03 at $95 \% \mathrm{Cl}, \mathrm{p}=0.09$ and had fewer laboratory confirmations compared to the participants taking the placebo $\mathrm{RR}=0.54+/$ 0.34 to 0.84 at $95 \%, p=0.007$ (Goodall, Granados \& Luinstra 2014).

The COVID-19 pandemic has raised the critical question of the correlation between VD health and COVID-19, which is increasingly being investigated. An SRMA found a retrospective cohort study took a total of 489 patients and separated the patients by their VD blood levels; multivariate analysis revealed the relative risk of testing positive for COVID-19 was $1.77+/-1.12$ -2.81 at $95 \%$ ( $p$-value $=0.02$ ) greater for patients with likely VDD levels than patients with appropriate VD levels (Meltzer, 2020). A Chinese SRMA from 2019 found that COVID-19 laboratory positive patients had higher incidences of low VD levels than COVID19 negative patients (Liu, Sun \& Wang 2021). An SRMA from ScienceDirect correlated the co-morbidities of COVID-19 that led to mortality have an inverse relationship on VD blood levels such as obesity and diabetes-explicitly explaining the high COVID-19 mortality rate in high-risk populations such as Blacks, Asians, and Hispanics (Nurshad, 2020). The final SRMA collected 14 different studies to conclude that low VD levels were significantly associated with an increased risk of COVID-19 infections as a qualitative synthesis and the pooled analysis. The stated odds ratio $(1.8+/-1.72$ to 1.88 at $95 \% \mathrm{Cl})$ meant the VDD population has an $80 \%$ chance of contracting COVID-19 (Teshome, Adane \& Girma 2021).

\section{CONCLUSION}

The "gold" standard of treatment and management for most VRIs is hydration and rest. No treatments like "antibiotics" for viruses, just a list of off-the-counter symptomatic medications. With the COVID-19 pandemic came the demise of healthcare infrastructures, vulnerable health populations, and public health esteem of many societies worldwide. Nonetheless, evidencebased medicine has presented an inversely causal relationship between VDD and VRIs. From all ages, low VD was associated with a higher risk of contracting a virus, higher duration of illness and could allow for longer transmissibility. The problem with most VD studies is the heterogeneity of most of the studies, SRMA and RCTs participants. Most have homogenous study samples; thus, the number of RCTs and large sample cohort studies is low and needs research. To ensure a proper prevention guide is set so that future generations can better attend to such biological atrocities.

Pilot RCTs are being conducted to see if VD or its metabolites could assist in treatment and management, first done by Goodell et al., who showed promising data in their RCTs showing VD 3 sufficient patients reported fewer clinical trials symptoms of VRIs and less laboratory confirmation from viral loads. A second piloted RCT with 50 ICU patients found that VD and its 


\section{Vitamin D Supplementation: A Potential Prevention Method against Contagious Viral Respiratory Illnesses}

metabolites significantly reduced the need for ICU treatment (Castillo, Costa \& Barrios 2020). An Oxford SRMAs of only 3 RCTs found the potential in VD and improving COVID-19 severity in hospitalized patients but needs more RCTs to prove its effects on mortality (Shah, Saxena \& Mavalankar 2021).

VD might not be the end-all-be-all, and however, it could be utilized as a preventative shield against communicable VRIs worldwide. Understanding the basics of VD effects on immunology is crucial to illustrate its proponents as a preventative health model. When VRI protective properties of VD are made aware to the public, the knowledge gained will spread to form a herd immunity-like shield. Proper VD intake to proper daily VD blood levels will decrease VRI prevalence and incidence, indirectly controlling the spread and decreasing infection duration.

Medical Professionals can be the gateway to this stream of VD information. For instance, different populations carry risk factors to VDD such as darker skin, elderly, overweight or obese, no fish or dairy in the diet, living closer to the poles of the earth, staying indoors, and applying certain sunscreens or sunblock. If general practitioners guide individuals and families to optimal VD levels, the public of many nations can fend off many varieties of VRIs (Martineau, 2017). Recommendations for RCTs and large cohorts surrounding VD and viral immunity should focus on reassessing, reevaluating, and updating current guidelines made by governmental agencies. To properly ask, when should VD levels start, how often should a general practitioner check them, and last new daily VD International units (IU) levels specifically for optimal IS Viral protection. VD recommendations were last made in 2014 by the USPTF, in the USA, for example. If successful, then places like the USA, and even globally, could be adequately protected and prevent another such worldwide viral pandemic no matter the origin.

\section{REFERENCES}

1) Abioye, A. I., Bromage, S., \& Fawz, W. (2021, January 1). Effect of micronutrient supplements on influenza and other respiratory tract infections among adults: a systematic review and meta-analysis. BMJ Global Health. https://gh.bmj.com/content/6/1/e003176

2) Castillo, M., Costa, L., \& Barrios, J. (2020, October 1). Effect of calcifediol treatment and best available therapy versus best available therapy on intensive care unit admission and mortality among patients hospitalized for COVID-19-19: A pilot randomized clinical study. ScienceDirect.

https://www.sciencedirect.com/science/article/abs/pii/S0960076020302764/?oRef=mixi

3) Coronavirus Update live (C. U. Live): 116,847,794 Cases and 2,594,982 Deaths from COVID-19-19 Virus Pandemic Worldometer. (2021, August 17 ${ }^{\text {th }}$ ). Worldometers. https://www.worldometers.info/coronavirus/

4) Goodall, E. C. (2014, May 19). Vitamin D3 and gargling for the prevention of upper respiratory tract infections: a randomized controlled trial. BMC Infectious Diseases. https://bmcinfectdis.biomedcentral.com/articles/10.1186/14712334-14-273\#citeas

5) Grant, W. B. (2020, April 2). Evidence that Vitamin D Supplementation Could Reduce Risk of Influenza and COVID-19-19 Infections and Deaths. MDPI. https://www.mdpi.com/2072-6643/12/4/988

6) Hernández, J. L., Nan, D., \& Fernandez-Ayala, M. (2020, October 27). Vitamin D Status in Hospitalized Patients with SARSCoV-2 Infection. OUP Academic.

https://academic.oup.com/jcem/advance-article/doi/10.1210/clinem/dgaa733/5934827

7) Huang, C., Wang, Y., \& Li, X. (2020, February 15). Clinical features of patients infected with 2019 novel coronavirus in Wuhan, China. ScienceDirect. https://www.sciencedirect.com/science/article/abs/pii/S0140673620301835

8) Jolliffe, D. A., Camargo Jr, C. A., \& Sluyter, J. D. (2021, May 1). Vitamin D supplementation to prevent acute respiratory infections: a systematic review and meta-analysis of aggregate data from randomized controlled trials. ScienceDirect. https://www.sciencedirect.com/science/article/pii/S2213858721000516

9) Laaksi, I. (2011, November 25). Vitamin D and respiratory infection in adults / Proceedings of the Nutrition Society. Cambridge Core. https://www.cambridge.org/core/journals/proceedings-of-the-nutrition-society/article/vitamin-d-andrespiratory-infection-in-adults/F0E08A242EBBE125087C8A7F65B1C6CC

10) Lemire, J. M. (1984, May). 1,25-dihydroxyvitamin D3 suppresses human $T$ helper/inducer lymphocyte activity in vitro. PubMed. https://pubmed.ncbi.nlm.nih.gov/3156926/

11) Liu, P. T. (2006, March 24). Toll-Like Receptor Triggering of a Vitamin D-mediated Human Antimicrobial Response. Science. https://science.sciencemag.org/content/311/5768/1770.abstract

12) Liu, N., Sun, J., \& Wang, X. (2021, March 1). Low vitamin D status is associated with coronavirus disease 2019 outcomes: a systematic review and meta-analysis. ScienceDirect.

https://www.sciencedirect.com/science/article/pii/S120197122032600X 
Vitamin D Supplementation: A Potential Prevention Method against Contagious Viral Respiratory Illnesses

13) Martineau, A. R. (2017, February 15). Vitamin D supplementation to prevent acute respiratory tract infections: systematic review and meta-analysis of individual participant data. The BMJ. https://www.bmj.com/content/356/bmj.i6583

14) Meltzer, D. O. (2020, September 3). Association of Vitamin D Status and Other Clinical Characteristics With COVID-19-19 Test Results. Infectious Diseases / JAMA Network Open / JAMA Network. https://jamanetwork.com/journals/jamanetworkopen/fullarticle/2770157

15) NIH, Office of Dietary Supplements. (2020, October 9). Office of Dietary Supplements - Vitamin D. Ods.Od.Nih.Gov. https://ods.od.nih.gov/factsheets/Vitamin\%20D-HealthProfessional/

16) Nurshad Ali, N. A. (2020, October). Role of vitamin D in preventing of COVID-19-19 infection, progression, and severity. ScienceDirect. https://www.sciencedirect.com/science/article/pii/S1876034120305311

17) Pham, H. (2019, August 21). Acute Respiratory Tract Infection and 25-Hydroxyvitamin D Concentration: A Systematic Review and Meta-Analysis. MDPI. https://www.mdpi.com/1660-4601/16/17/3020/htm

18) Jat K. R. (2017). Vitamin D deficiency and lower respiratory tract infections in children: a

a. systematic review and meta-analysis of observational studies. Tropical doctor, 47(1), 77-84. https://doi.org/10.1177/0049475516644141

19) Shah, K., Saxena, D., \& Mavalankar, D. (2021, January 24). Vitamin D supplementation, COVID-19 and disease severity: a meta-analysis. OUP Academic. https://academic.oup.com/qjmed/article/114/3/175/6118232?login=true

20) Sharifi, A., Vahedi, H., Nedjat, S., Rafiei, H., \& Hosseinzadeh-Attar, M. J. (2019, July). Effect of single-dose injection of vitamin $D$ on immune cytokines in ulcerative colitis patients: a randomized placebo-controlled trial. PubMed. https://pubmed.ncbi.nIm.nih.gov/31274211/

21) Sizar, O (2021, January 3). Vitamin D Deficiency. PMC Library. Retrieved 03/03/2021, https://www.ncbi.nlm.nih.gov/books/NBK532266/

22) Spritzler, F. (2018, July 23). 8 Signs and Symptoms of Vitamin D Deficiency. Healthline. https://www.healthline.com/nutrition/vitamin-d-deficiency-symptoms

23) Teshome, A., Adane, A., \& Girma, B. (2021, March 5). The Impact of Vitamin D Level on COVID-19-19 Infection: Systematic Review and Meta-Analysis. Frontiers.

https://www.frontiersin.org/articles/10.3389/fpubh.2021.624559/full?utm_source=STWT\&utm_medium=SNET\&utm_c ampaign=ECO_FPUBH_XXXXXXXX_auto-dlvrit

\section{APPENDICES}

Appendix 1

\section{Abbreviations}

\begin{tabular}{|c|c|}
\hline Cellular Adaptive Immunity & CAI \\
\hline Cellular Innate Immunity & $\mathrm{Cll}$ \\
\hline Intensive Care Unit & $\mathrm{ICU}$ \\
\hline Immune System & IS \\
\hline Metabolites & $\mathrm{VD}_{2}+\mathrm{VD}_{3}$ \\
\hline Narrative Review & NR \\
\hline Randomized Control Trials & $\mathrm{RCT}$ \\
\hline SARS-Coronavirus-2/COVID-19, & COVID \\
\hline Systematic Review and Meta-Analysis & SRMA \\
\hline T helper 1 Cells & $\mathrm{Th}_{1}$ \\
\hline T helper 2 Cells & $\mathrm{Th}_{2}$ \\
\hline United States Preventative Task Force & USPTF \\
\hline Viral Respiratory Illnesses & VRI \\
\hline Vitamin D/Calciferol & VD \\
\hline Vitamin D/Calciferol Deficiency & VDD \\
\hline Vitamin D2/Ergocalciferol & $\mathrm{VD}_{2}$ \\
\hline Vitamin $\mathrm{D}_{3} /$ Cholecalciferol/Calcitriol & $\mathrm{VD}_{3}$ \\
\hline Vitamin $D_{3} /$ Cholecalciferol/Calcitriol Deficiency & $\mathrm{VD}_{3} \mathrm{D}$ \\
\hline
\end{tabular}

